\title{
Confinement and Pseudoscalar Glueball Spectrum in the $2+1$ D QCD-Like Theory from the Non-Susy D2 Brane
}

\author{
Adrita Chakraborty ${ }^{1, *}$ \\ ${ }^{1}$ Department of Physics, Indian Institute of Technology Kharagpur, Kharagpur, India-721302
}

\begin{abstract}
We study two important properties of 2+1D QCD, namely confinement and Pseudoscalar glueball spectrum, using holographic approach. The confined state of the bounded quark-antiquark pair occurs in the self-coupling dominated nonperturbative regime, where the free gluons form the bound states, known as glueballs. The gauge theory corresponding to low energy decoupled geometry of isotropic non-supersymmetric D2 brane, which is again similar to the 2+1D YM theory, has been taken into account but in this case the coupling constant is found to vary with the energy scale. At BPS limit, this theory reduces to supersymmetric YM theory. We have considered NG action of a test string and calculate the potential of such confined state located on the boundary. The QCD flux tube tension for large quark-antiquark separation is observed to be a monotonically increasing function of running coupling. The mass spectrum of Pseudoscalar glueball is evaluated numerically from the fluctuations of the axion in the gravity theory using WKB approximation. This produces the mass to be related to the string tension and the levels of the first three energy states. The various results that we obtained quite match with those previously studied through the lattice approach.
\end{abstract}

\section{Introduction}

Unlike the four dimensional theory, the three dimensional Yang-Mills theory having the dimension-full coupling $g_{\mathrm{YM}}^{2}$ is not classically scale-invariant. In spite of this difference, the lower dimensional Yang-Mills theory has some interesting similarities with the $3+1 \mathrm{di}-$ mensional counterpart, e.g., ultraviolet freedom, infra-red slavery, energy-scale dependent running effective coupling, confinement, glueball mass spectrum etc. Because of this, the $2+1$ dimensional pure Yang-Mills theory has been studied widely in various approaches [1-5]. So, at low energy scale the color confinement potential has been found to have the well-known linear dependency on the spatial separation between charges, $r$, i.e., $V(r) \sim \sigma r$. $\sigma$ represents the QCD flux-tube tension. For large $N_{c}, 2+1$ dimensional pure YM theory is theoretically found to have QCD flux-tube tension [6],

$$
\sigma=g_{\mathrm{YM}}^{4} \frac{N_{c}^{2}-1}{8 \pi}
$$

This confinement indicates strong self-coupling dominated regime where the free gluons form the bound states, called glueballs. The presence of such bound state can be observed from

\footnotetext{
*e-mail: adimanta09@iitkgp.ac.in
} 
its discrete energy spectrum or mass gap in the confined phase. Here we study these two properties of the $2+1$ dimensional non-perturbative QCD with the holographic approach.

In the planar or large $N_{c}$ limit of the AdS/CFT duality, the string coupling $g_{s}$ is so small that the gravity theory is perturbative. This eventually results in very small YM coupling $\left(g_{Y M}^{2} N_{c} \sim g_{s} \rightarrow 0\right)$. But to ensure of the strong coupling nonperturbative behaviour in the gauge theory side the number of D-branes, $N_{c}$, living on the boundary of the bulk is taken to be $N_{c} \rightarrow \infty$. This duality has been also proved to be an effective technique to unravel non-perturbative non-conformal QCD-like theories by dragging it to some gravity dual on non-AdS background. Despite the absence of asymptotic freedom due to strong YM coupling, many other non-perturbative behaviours of QCD such as confinement, chiral symmetry breaking, scattering cross-section, glueballs, thermal phase transition, etc., can be studied from holography[1].

Here, we consider non-supersymmetric non-BPS D2 brane solutions of the type-II supergravity [7] as the gravity theory dual to a $2+1$ dimensional Yang-Mills gauge theory. As the supersymmetry is broken the solutions have more parameters than the usual BPS ones. These parameters are related to the coupling and the UV fixed point, thereby making the dual nonconformal theory QCD-like. Here we consider a stack of $N_{c}$ number of the non-susy, isotropic D2 branes where the background dilaton field $\phi$ and consequently the effective coupling of 2+1D QCD-like Yang-Mills theory vary with the length scale of the bulk theory. The low energy decoupling limit is considered to find the near brane geometry of the non-susy D2 brane. Then we configure a probe open string containing the quark-antiquark pair at its extreme endpoints and employed the holographic understandings of the thermal expectation value of minimal Wilson loop to compute the linear $Q \bar{Q}$ confinement potential. The presence of confinement in the low energy scale triggered us to evaluate the mass spectrum of glueball states. We have presented hither, the mass spectrum of Pseudoscalar glueballs in the 2+1D pure YM theory from the Schrödinger-like field equation of the axion's fluctuation using the WKB approximation. The mass spectrum is evaluated numerically but the restrictions in the WKB method constrained us in the lower states only.

\section{Gravity Background}

In this section we will discuss the non-susy D2 brane solution and its decoupled geometry at low energy limit.

\subsection{Non-Susy D2 Brane}

Besides the supersymmetric BPS brane, there is a similar sector of the non-supersymmetric Dp brane solutions of the type-II supergravity containing unequal ADM mass and charge[7]. In the BPS or extremal limit, the number of free parameters associated to non-susy branes reduces so that they merge into BPS branes of same dimension.

The non-susy D2 brane solution assumes a form in the String frame as [8].

$$
\begin{gathered}
d s^{2}=F(\rho)^{-\frac{1}{2}} G(\rho)^{\frac{\beta}{4}+\frac{\delta}{4}}\left(-d t^{2}+\sum_{i=1}^{2}\left(d x^{i}\right)^{2}\right)+F(\rho)^{\frac{1}{2}} G(\rho)^{\frac{1}{5}-\frac{\beta}{4}+\frac{\delta}{4}}\left(\frac{d \rho^{2}}{G(\rho)}+\rho^{2} d \Omega_{6}^{2}\right) \\
e^{2 \phi}=e^{2 \phi_{0}} F(\rho)^{\frac{1}{2}} G(\rho)^{\delta-\frac{\beta}{4}}, \quad F_{[6]}=Q \operatorname{Vol}\left(\Omega_{6}\right)
\end{gathered}
$$

where

$$
F(\rho)=G(\rho)^{\gamma} \cosh ^{2} \theta-\sinh ^{2} \theta, \quad G(\rho)=1+\frac{\rho_{2}^{5}}{\rho^{5}}
$$


are the harmonic functions introduced due to the relaxation in BPS condition. The $(2+1)$ dimensional worldvolume is defined with the coordinates $\left(t, x^{1}, x^{2}\right)$ whereas the seven dimensional transverse space is defined by the spherical coordinates $\left(\rho, \Omega_{6}\right) . \rho$ is the radial coordinate perpendicular to the worldvolume thereby representing the energy scale of the theory as $\sim 1 / \rho$. The brane is taken to be located at the singularty $\rho=0$. In this geometry, the dilaton field is quite vividly found to be a non-trivial function of the energy scale of the gravity theory. Henceforth, the effective coupling of the theory also varies with the energy scale. Though the geometry contains six parameters only three out of them are independent, namely, mass parameter $\rho_{2}$, charge parameter $\theta$ and dilatonic parameter $\delta$. The definitions of the harmonic functions $G(\rho)$ and $F(\rho)$ in the range $0<\rho<\infty$ suggests that the value of the parameter $\delta$ should be bounded in the range $-\frac{8}{5} \leq \delta \leq \frac{8}{5}$.

\subsection{Decoupled Geometry}

Akin to the BPS branes, the non-susy branes also decouple from the bulk theory at low energy limit [8]. However, these geometries are not AdS implying about their non-conformality. From the understanding of gauge/gravity duality, this non-AdS gravity theory corresponds to the non-conformal gauge theory defined in the worldvolume of the brane. As the fundamental string length $\ell_{s} \rightarrow 0$ at the low energy limit, we consider a scaling of the bulk radial coordinate accordingly to get a finite scale in near brane regime and it is given as

$$
\rho=\alpha^{\prime} u, \rho_{2}=\alpha^{\prime} u_{2}, \sinh ^{2} \theta=\frac{L}{\gamma u_{2}^{5} \alpha^{\prime 2}}
$$

where $u$ having mass dimension +1 defines the new radial coordinate of the decoupled geometry as well as the energy scale of the theory. $u_{2}$ is a constant parameter that comes into play due to the non-conformal structure of the theory. The other quantity $L$ is defined as $L=3 \pi^{2} g_{\mathrm{YM}}^{2} N_{c}$, where $g_{\mathrm{YM}}^{2}$ is the coupling in $2+1$ dimensional Yang-Mills theory. Thus using the scaling (3), we can rewrite the decoupled geometry of the background (1) as follows.

$$
\begin{aligned}
d s^{2} & =\alpha^{\prime}\left[\sqrt{\frac{\gamma u_{2}^{5}}{L}} F(u)^{-\frac{1}{2}} G(u)^{\frac{\delta}{4}+\frac{\beta}{4}}\left(-d t^{2}+\sum_{i=1}^{2}\left(d x^{i}\right)^{2}\right)+\sqrt{\frac{L}{\gamma u_{2}^{5}}} F(u)^{\frac{1}{2}} G(u)^{\frac{1}{5}+\frac{\delta}{4}-\frac{\beta}{4}}\left(\frac{d u^{2}}{G(u)}+u^{2} d \Omega_{6}^{2}\right)\right], \\
e^{2 \phi} & =\frac{g_{\mathrm{YM}}^{4}}{4} \sqrt{\frac{L}{\gamma u_{2}^{5}}} F(u)^{\frac{1}{2}} G(u)^{\delta-\frac{\beta}{4}} F_{[6]}=5 L \alpha^{\prime 3} \operatorname{vol}\left(\Omega_{6}\right), F(u)=G(u)^{\gamma}-1, G(u)=1+\frac{u_{2}^{5}}{u^{5}}
\end{aligned}
$$

In these decoupled forms of the harmonic functions, $\gamma$ can not be zero. So the allowed range of $\delta$ is now modified to $-\frac{8}{5}<\delta<\frac{8}{5}$. In this non-conformal decoupled geometry, the curvature of the $7 \mathrm{~d}$ transverse space is small enough to validate the holographic calculation in the low energy scale. Now the effective gauge coupling $\lambda^{2}$ can be written from the dilaton field following the standard relation $e^{\phi} \sim \frac{\lambda^{\frac{5}{2}}}{N_{c}}[2]$.

$$
\lambda^{2}=\frac{1}{\left(6 \pi^{2}\right)^{\frac{4}{5}}} \gamma^{\frac{1}{5}}\left(\frac{L}{u_{2}}\right) F(u)^{\frac{1}{5}} G(u)^{\frac{2}{5} \delta-\frac{\beta}{10}}
$$

which evidently varies with the energy scale $u$ and the parameter $\delta$. Thus in the corresponding gauge theory, we have the running coupling similar to QCD. So the theory on the brane is a $(2+1)$ dimensional QCD-like theory without flavour. 
Like the QCD theory, the effective gauge coupling $\lambda^{2}$ in (5) decays with the increasing energy scale $u=\epsilon u_{2}$ which is shown in the Figure 1. Again, at the fixed energy scale $u$, $\lambda^{2}$ is also found to increase monotonically with the increasing value of $\delta$. According to holographic point of view, the type-IIA supergavity describes the non-perturbative gauge theory for the range of $u \sim u_{2}$, only if

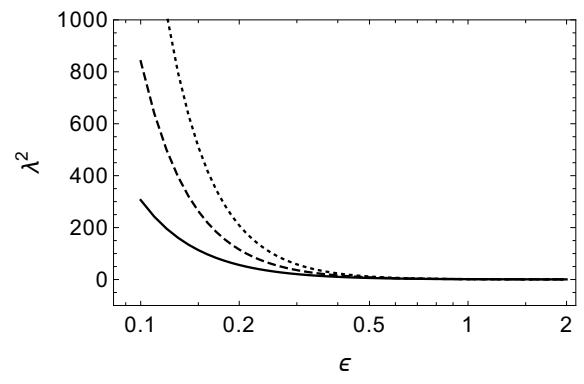

Figure 1: Dependence of $\lambda^{2}$ on the energy scale

$\frac{1}{\gamma^{1 / 5}} g_{\mathrm{YM}}^{2} N_{c}^{\frac{1}{5}} \leq u_{2} \leq g_{\mathrm{YM}}^{2} N_{c}$. At $u \gg u_{2}$, the geometry (4) reduces to the decoupled geometry of the BPS D2 brane with the effective coupling $\lambda^{2} \sim \frac{g_{\mathrm{YM}}^{2} N_{c}}{u}$. On the other hand, at the limit $u \ll u_{2}$, the effective coupling $\lambda^{2}$ becomes very large for the given range of $\delta$ and we have to uplift our theory in the worldvolume of 11d M2 brane to keep supergravity approximation justifiable.

\section{Confinement}

The establishment of the confinement property has indulged an open string connecting the quark antiquark along its length on the boundary and hanging towards the singularity $u=0$ in the bulk. Employing the holographic prescription of the minimally swept out Wilson Loop and taking the minimised components of the metric in the NG action, we reach the binding potential of the $Q \bar{Q}$ pair for the maximum separation $\Delta x$ between them as

$$
V=\frac{u_{2}^{\frac{5}{2}}}{2 \pi \sqrt{L}}\left[\frac{5}{8} \delta^{2}-\frac{3}{5}\right]^{\frac{1}{4}} \sum^{\frac{5 \delta}{16 \gamma}} \Delta x
$$

The $Q \bar{Q}$ potential is linearly proportional to this spatial length and therefore confirms of the confinement property of the theory herein. The coefficient multiplied with $\Delta x$ has dimension of string tension, which is identified as the QCD string tension $\sigma$ and is expressed as [9]

$$
\frac{\sigma}{g_{\mathrm{YM}}^{4} N_{c}^{2}}=\frac{9 \pi^{3}}{2}\left(\frac{u_{2}}{L}\right)^{\frac{5}{2}}\left(\frac{5}{8} \delta^{2}-\frac{3}{5}\right)^{\frac{1}{4}} \Sigma^{\frac{5 \delta}{16 \gamma}}
$$

Moreover, the maximum $Q \bar{Q}$ separation $\Delta x$ is achieved when the vertical distance of the turning point of the probe string from the singularity becomes minimum. This stable configuration can be obtained at

$$
u_{m}=\frac{u_{2}}{\left(\Sigma^{1 / \gamma}-1\right)^{1 / 5}} \text { where, } \Sigma=\frac{5 \delta+4 \gamma}{5 \delta-4 \gamma}
$$

It is clear from the above turning point that it does not exist for the whole parametric range. Since $\gamma$ is always positive, it exists only if $\Sigma>1$ or $5 \delta+4 \gamma>5 \delta-4 \gamma$. Then the allowed parametric regime shrinks to $\frac{2 \sqrt{6}}{5}<\delta<\frac{8}{5}$. Beyond this range, the test string goes into the singularity without any valid turning point. Here we have taken $u_{2}=g_{\mathrm{YM}}^{2} N_{c}$ which makes the ratio $\frac{\sqrt{\sigma}}{g_{\mathrm{YM}}^{2} N_{c}}$ independent of $g_{\mathrm{YM}}^{2} N_{c}$. The variation of the ratio $\frac{u_{m}}{u_{2}}$ with the parameter $\delta$ in this specified parametric range is depicted in the Figure 2. The nature of the QCD string 

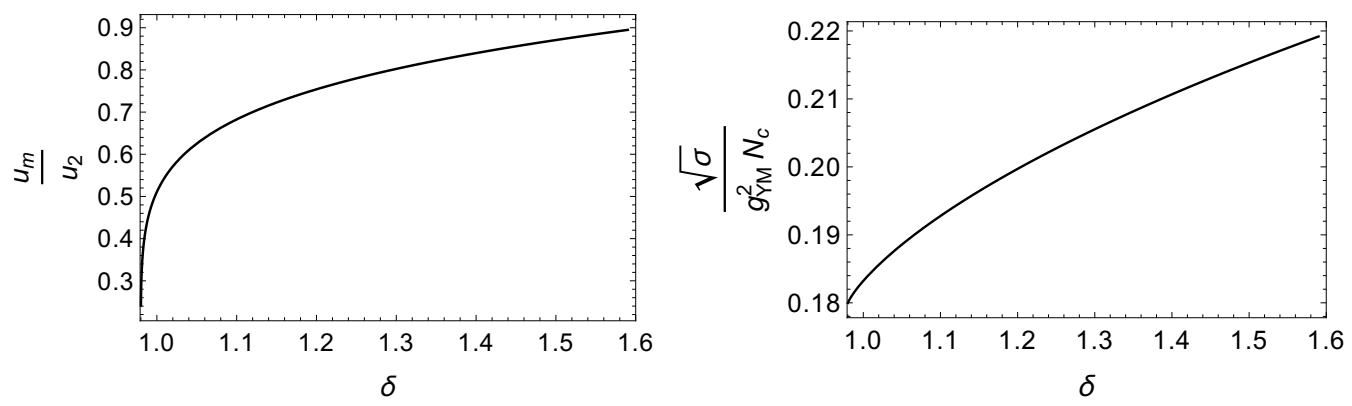

Figure 2: Dependence of the ratio $\frac{u_{m}}{u_{2}}$ with $\delta$ and the $\delta$-dependence of QCD string tension

Table 1: Values of $\sqrt{\sigma}$ calculated from (7) at $u_{2}=g_{\mathrm{YM}}^{2} N_{c}$.

\begin{tabular}{|c|c|c|c|c|c|c|c|}
\hline$\delta$ & 0.98 & 1.08 & 1.18 & 1.28 & 1.38 & 1.48 & 1.58 \\
\hline$\frac{\sqrt{\sigma}}{g_{\mathrm{YM}}^{2} N_{c}}$ & 0.1799 & 0.1912 & 0.1984 & 0.2044 & 0.2097 & 0.2144 & 0.2187 \\
\hline
\end{tabular}

tension $\sigma$ has been observed in Table 1 [9] by varying the background parameter $\delta$ at the entirely nonperturbative regime of the theory that is ensured by taking $u_{2}=g_{Y M}^{2} N_{c}$. Now the theoretical value of $\sqrt{\sigma}$ is 0.1995 for pure Yang-Mills theory. In lattice calculation [5], the value is 0.1975 . Both of these values are evaluated at $N_{c}=\infty$. There are also some recent observations[10], both in lattice and theoretical methods, where the estimated string tension approximately meets these values. Here for various $\delta, \sqrt{\sigma} / g_{\mathrm{YM}}^{2} N_{c}$ varies in the range $0.19 \pm 0.02$ for large $N_{c}$. So our calculation fairly matches with those previous results. It is also clear from Table 1 that the string tension is a monotonically increasing function of $\delta$ which is also depicted in Figure 2. As we have seen previously that $\lambda^{2}$ increases with $\delta$, the string tension increases with the increasing effective coupling $\lambda^{2}$. This dependence of the string tension on the effective coupling strengthens its QCD-like nature and indicates towards the confinement property of the pure 2+1D YM theory. The existence of the confinement alludes the theory to be in the self-coupling dominated non-perturbative regime. Now, if we take $u_{2} \rightarrow 0$, to get a finite turning point in (8) we need to take $\delta \rightarrow \frac{8}{5}$ so that the string tension in (7) takes the form $\frac{\sigma}{g_{\mathrm{YM}}^{4} N_{c}^{2}}=\frac{9 \pi^{3}}{2}\left(\frac{u_{2}}{L}\right)^{\frac{5}{2}}$, which goes to zero as $u_{2} \rightarrow 0$. Thus in the BPS limit, the gravity background (4) reduces to the decoupled geometry of the BPS D2 brane and the dual gauge theory becomes $\mathcal{N}=8$ deconfined super-Yang-Mills theory in $2+1$ dimensions.

\section{Mass Spectrum of Pseudoscalar $0^{-+}$Glueballs}

In the low energy self-coupling dominated regime, the confinement nature of the theory brings the gluons closer and makes the bound states - glueball. Although, the glueball is not observed experimentally till date, the mass spectrum of the glueballs has been calculated theoretically [11] and in lattice QCD [5, 12, 13]. Similar studies have been conducted for the glueballs in the three dimensional QCD by implementing both the lattice $[5,14]$ and holographic approaches [2]. In this section, we have explored the pseudoscalar glueball spectrum in holographic 3D QCD by incorporating the non-susy brane solutions. The pseudoscalar glueball mass is associated with the axion field of the bulk. As the axion field couples minimally with the background, the fluctuation does not change the background metric. Starting 
with the linearised field equation for axion fluctuation $\chi=f(u) e^{i k_{a} x^{a}}$, where $a=0,1,2$ and $k_{a} k^{a}=-M^{2}$, we have computed the mass spectrum $M$ for the pseudoscalar light glueballs by means of WKB approximation. For the sake of simplicity, we demand the fluctuation field $\chi$ to be symmetric on the six dimensional transverse sphere of (4), polarised along the brane worldvolume, and also a function of $u$. So the potential is almost flat and positive in the whole range except $y_{-} \leq y \leq y_{+}$. Thus it forms a small potential well with boundaries at $y_{-}$and $y_{+}$. The depth of the well is regulated by $\delta$ and the mass $m$. Thus using WKB approximation we can estimate $m$ for given $\delta$. Here due to the technical restrictions of WKB aproximation, we calculate first few energy states of the spectrum.

Table 2: Mass $M_{0^{-+}}$and $\sqrt{\sigma}$ in unit of $\sqrt{u_{2}^{3} / L}$ and $10^{-3} L$ respectively, for various $\delta$

\begin{tabular}{|c|c|c|c|c|}
\hline$\delta$ & $M_{0^{-+}}$ & $M_{0^{-+}}^{*}$ & $M_{0^{-+}}^{* *}$ & $\sqrt{\sigma}$ \\
\hline 0.98 & 6.15797 & 9.70152 & 12.9685 & 6.07865 \\
1.08 & 6.25563 & 9.86602 & 13.2028 & 6.45687 \\
1.18 & 6.35031 & 10.0252 & 13.4294 & 6.70183 \\
1.28 & 6.44245 & 10.1799 & 13.6493 & 6.90446 \\
1.38 & 6.53266 & 10.3310 & 13.8638 & 7.08193 \\
1.48 & 6.62202 & 10.4803 & 14.0746 & 7.24190 \\
1.58 & 6.71494 & 10.6346 & 14.2905 & 7.38863 \\
\hline \hline
\end{tabular}

In the Table 2, we have listed the dimensionless scaled mass $m$ for different $\delta$, where the actual mass $M_{0^{-+}}$is $m \sqrt{u_{2}^{3} / L}$ [9]. The mass values have been found to increase with the increasing values of $\delta$. Also, we have already seen the effective coupling $\lambda$ as an increasing function of $\delta$. Therefore combinatorially the increasing $\delta$ causes increase in the self-coupling of the gluon fields. Due to this rising of self-coupling, the glueballs become more massive in the sea of gluon. Therefore in this aspect, the calculated mass values show the consistency with the nature of QCD-like theories. We also evaluate the ratio $M_{0^{-+}} / \sqrt{\sigma}$ for $n=0,1,2$ states in the complete non-perturbative region [9] and this put forth an emperical relation of pseudoscalar glueball masses to the QCD flux-tube tension as

$$
M_{0^{-+}} \approx \frac{10^{3}}{6 \sqrt{3} \pi^{3}}(n+2) \sqrt{\sigma} \approx 3(n+2) \sqrt{\sigma}
$$

Therefore the mass-to-tension ratio is almost constant for a particular energy state, which is one of the similarities of QCD3 with the four dimensional QCD. From this empirical relation the ratio of the mass of the first excited state to that of the ground state mass is 1.5. To date we have not found the enough data for the full pseudoscalar spectrum. But the complete spectrum is available in four dimensions. So now we first take a comparative look on those results. In QCD4 [12], $M_{0^{++}}^{*} / M_{0^{++}}=1.54$ and $M_{0^{-+}} / M_{0^{++}}=1.50$. On the other hand, in QCD3 these two ratios are respectively 1.5061 and 2.148 in lattice calculation [5]. However in Holographic approach $[2,15], M_{0^{++}}^{*} / M_{0^{++}}=1.72$ in QCD3. So it seems that the glueballs are comparatively more massive in $2+1$ dimensions. Therefore we can expect the ratio $M_{0^{-+}}^{*} / M_{0^{-+}}$which is found to be 1.40 in lattice calculation [12] and 1.46 in holography [15] as QCD4 acquires a higher value in the $2+1$ dimensional theory. Here we get the ratio in the range 1.575-1.583. Due to the behavioural consistencies of this mass spectrum with the QCD, we can expect that these mass values are also consistent with the pure Yang-Mills theory in $2+1$ dimensions. 


\section{Conclusion}

Here we have obtained the string tension and the pseudoscalar glueball spectrum in $2+1 \mathrm{di}$ mensional strongly coupled gauge theory in the entirely non-perturbative region by assuming $u_{2} \sim g_{\mathrm{YM}}^{2} N_{c}$ from the non-susy D2 brane of type-IIA supergravity. The gravity background has two free parameters - the dimensionless $\delta$ and the dimension-full $u_{2}$. $u_{2}$ controls the nature of the gauge theory and $\delta$ measures the zero temperature gluon-condensate in that gauge theory. The string tension $\sigma$ is found to depend on the dilatonic background parameter $\delta$. It can be found that for a particular value of $\delta$, the string tension matches exactly with the results of the pure Yang-Mills theory in $2+1$ dimensions. Along with this, the effective gauge coupling $\lambda^{2}$ is monotonically decreasing function of the energy scale $u$, thereby credibly supporting for the confinement properties achieved in the considered $2+1 \mathrm{D}$ pure YM-like theory. Here we have elucidated on the numerical computation of the mass spectrum of the pseudoscalar glueball using WKB approximation. The spectrum given in the Table 2 is found to have consistent nature with QCD. The masses increase with the increasing effective coupling of the theory. However the coupling dependence of the spectra has not been shown explicitly. The ratio of the mass to the square root of the string tension has been found to be constant which is also analogous to the four dimensional QCD. All of these similarities stem for the assertion that the holographic dual of the non-susy D2 brane in the considered parametric range is the $2+1$-dimensional QCD-like theory. But the non-AdS geometrical structure of the gravity background has made it hard to find the complete picture of this QCD-like gauge theory. Due to its complicated mathematical structure, we have evaluated the mass spectrum numerically using WKB approximation. It will be interesting to find the mass spectrum analytically. In this way, we will be able to find the analytic expressions of the masses showing explicit dependence on various parameters. The thermal evolution of the string tension and the glueball spectrum in the $2+1$ dimensional gauge theory is also an interesting topic to study in non-susy brane background of type-II supergravity.

\section{References}

[1] E. Witten, Adv. Theor. Math. Phys. 2, 505-532 (1998)

[2] O. Aharony, S. S. Gubser, J. M. Maldacena, H. Ooguri and Y. Oz, Phys. Rept. 323, 183-386 (2000)

[3] D. Karabali and V. P. Nair, Nucl. Phys. B 464, 135-152 (1996); D. Karabali and V. P. Nair, Int. J. Mod. Phys. A 12, 1161-1172 (1997); D. Karabali, C. j. Kim and V. P. Nair, Phys. Lett. B 434, 103-109 (1998)

[4] M. Teper, Phys. Lett. B 311, 223-229 (1993);

[5] M. J. Teper, Phys. Rev. D 59, 014512 (1999)

[6] V. P. Nair, Nucl. Phys. B Proc. Suppl. 108, 194-200 (2002)

O. Philipsen, M. Teper and H. Wittig, Nucl. Phys. B 469, 445-472 (1996)

[7] J. X. Lu and S. Roy, JHEP 02, 001 (2005)

[8] K. Nayek and S. Roy, JHEP 03, 102 (2016)

[9] A. Chakraborty and K. Nayek, Phys. Rev. D 103, 066001 (2021)

[10] M. Frasca, Eur. Phys. J. C 77, no.4, 255 (2017); R. Lau and M. Teper, JHEP 10, 022 (2017)

[11] N. R. Constable and R. C. Myers; JHEP 11, 020 (1999), C. Csaki and M. Reece; JHEP 05, 062 (2007) R. de Mello Koch, A. Jevicki, M. Mihailescu and J. P. Nunes, Phys. Rev. D 58, 105009 (1998)

[12] C. J. Morningstar and M. J. Peardon, Phys. Rev. D 60, 034509 (1999); 
[13] Y. Chen, A. Alexandru, S. J. Dong, T. Draper, I. Horvath, F. X. Lee, K. F. Liu, N. Mathur, C. Morningstar, M. Peardon, S. Tamhankar, B. L. Young and J. B. Zhang, Phys. Rev. D 73, 014516 (2006)

[14] M. Teper, Phys. Lett. B 397, 223-228 (1997); O. Philipsen, M. Teper and H. Wittig, Nucl. Phys. B Proc. Suppl. 53, 626-628 (1997)

[15] C. Csaki, H. Ooguri, Y. Oz and J. Terning, JHEP 01, 017 (1999) 